\title{
Mapping of grain alkali digestion trait using a Cheongcheong/Nagdong doubled haploid population in rice
}

\author{
Hak Yoon Kim $\cdot$ Kyung-Min Kim
}

Received: 16 January 2016 / Revised: 11 February 2016 / Accepted: 15 February 2016

(C) Korean Society for Plant Biotechnology

\begin{abstract}
We performed a molecular marker-based analysis of quantitative trait loci for traits that determine the quality of appearance of grains using 120 doubled haploid lines developed by anther culture from the $F_{1}$ cross between 'Cheongcheong' (Oryza sativa L. ssp. Indica) and 'Nagdong' (Oryza sativa L. ssp. Japonica). We therefore calculated the alkali digestion value (ADV), used to indirectly measure gelatinization temperature, to evaluate the quality of cooked rice in 2013 and 2014. The ADV score of frequency distribution was higher milled rice than brown rice. In total, nine different quantitative trait loci (QTLs) were found on 5 chromosomes in 2013 and 2014. Also, chromosome 5, 8 were detected over two years. We conclude that selected molecular markers from this QTL analysis could be exploited in future rice quality. In conclusion, we investigated ADV of brown and milled rice in CNDH population. This study found nine QTLs related to the ADV of brown and milled rice. The detected one marker can be used to select lines with desirable eating-quality traits because ADV is closely associated with the eating quality of cooked rice. Therefore, it will be useful to collect resources and distinguishable in many varieties for rice breeding program.
\end{abstract}

Keywords Alkali digestion value, QTL, Rice, Markerassisted selection

H. Y. Kim

Department of Global Environment, Keimyung University, Daegu, 42601, Korea

K.-M. Kim ( $\triangle)$

Division of Plant Biosciences, School of Applied Biosciences, College of Agriculture \& Life Science, Kyungpook National

University, Daegu, 41566, Korea

e-mail: kkm@knu.ac.kr

\section{Introduction}

In recent years, rice consumption in the Republic of Korea has seen a decrease in response to economic growth and westernization of dietary habits. To increase rice consumption and prepare for the opening of Korea's rice market internationally, the production of high-quality rice is necessary. The digestibility and taste of rice is related to rice gelatinization, which is affected by numerous factors, including amylose content $(\mathrm{Hu}$ et al. 2004). In Korea, cultivars with relatively low gelatinization temperatures are typically preferred. This characteristic can be measured indirectly using the alkali digestion value (ADV), which also functions to score the taste of cooked rice (Juliano 1985, 2003; Lee et al. 2000; Kwak 2009).Previous research has found that traits related to cooking quality were mainly controlled by genetic effects, but interactions between the genotype and the environment were also present (Shi et al. 1997). Furthermore, a major gene controlling amylose content (AC) was found to be linked with a gene involved in the alkali spreading score (Mckenzie and Rutger 1983). In rice breeding programs, using DNA markers to select promising individuals in early generations can improve efficiency, because the indirect evaluation of eating quality is possible through genotyping. Recently, advances in molecular technology have resulted in the development of DNA markers related to major quantitative trait loci (QTL). If the markers related to a major gene are employed to select for a polygenic trait, selection efficiency in breeding can be improved. Because eating-quality traits in rice are polygenic, it is especially necessary to focus our research on marker assist selection (MAS) associated with QTLs and the accumulation of various DNA markers related to desirable traits (Xiao et al. 1996; Lee et al. 2000). Therefore, we investigated alkali digestion values (ADV) in rice by analyzing QTLs to detect DNA markers associated with alkali digestion. We conducted our experiments one doubled haploid (DH) population, derived from a cross between 'Cheongcheong' and 'Nagdong' strains. 


\section{Materials and Methods}

Plant materials

Through anther culture, we developed $120 \mathrm{DH}$ lines, using an $F_{1}$ population derived from a 'Cheongcheong' and 'Nagdong' cross (hereafter, the CNDH line). 'Cheongcheong' is a indica-type while 'Nagdong' is a japonica-type (Fig. 1). In 2013 and 2014, the plants were cultivated and harvested at the experimental paddy field of Kyungpook National University in Gunwi, North Gyeongsang Province, Korea Republic. The population was transplanted at $30 \times 15 \mathrm{~cm}$ spacing. They were fertilized with $\mathrm{N}-\mathrm{P}_{2} \mathrm{O}_{5}-\mathrm{K}_{2} \mathrm{O}$ applied at 9-4.5-5.7 kg/10a, according to the Rural Development Administration (RDA) standard cultivation practice.

Evaluation of alkali digestion value

The evaluation of ADV was performed four times, using the following procedure. Prior to evaluation, rice grains were hulled and milled using a rice huller (SYTH-88, Ssangyong Machinery Industry Co., Ltd.) and a rice milling machine

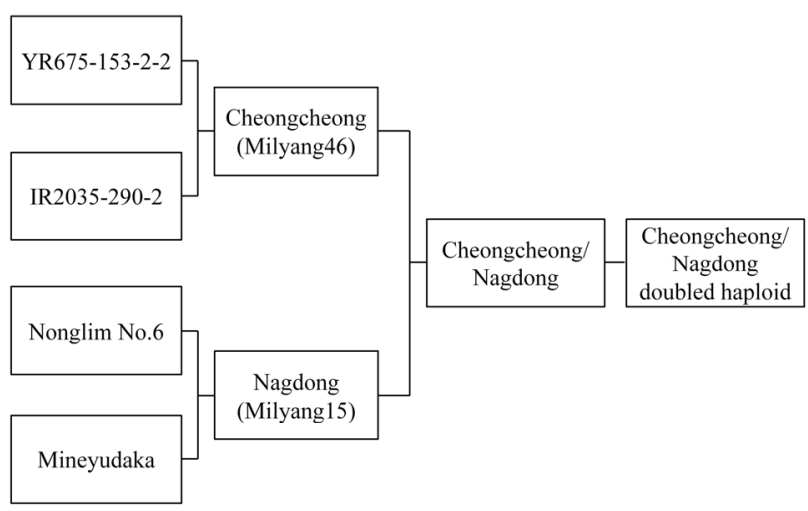

Fig. 1 Genealogical diagram of the Cheongcheong/Nagdong doubled haploid population. Cheongcheong is a Tongil-type rice cultivar from an indica and japonica cross, while Nagdong is a japonicatype rice cultivar. The doubled haploid population was developed using anther culture

Table 1 Criteria for alkali digestion value of rice from the International Rice Research Institute

\begin{tabular}{cl}
\hline Score & \multicolumn{1}{c}{ Alkali digestion value } \\
\hline 1 & Grain not affected \\
2 & Grain swollen \\
3 & Grain swollen, color incomplete or narrow \\
4 & Grain swollen, color complete and wide \\
5 & Grain split or segmented, color complete and wide \\
6 & Grain dispersed, merging with collar \\
7 & Grain completely dispersed and disintegrated \\
\hline
\end{tabular}

(Pearlest, Kett Electric Laboratory). Damaged grains, red grains, green grains and broken grains were removed from the brown rice and milled rice before testing. Brown and milled rice were placed on a petri dish $(90 \times 15 \mathrm{~mm})$ containing 20 $\mathrm{mL}$ of $1.4 \% \mathrm{KOH}$ solution, in separate rows of four grains each. The KOH solution consisted of $16.47 \mathrm{~g}$ of $85 \% \mathrm{KOH}$ powder dissolved into $1 \mathrm{~L}$ water. The grains were left undisturbed for 18 hours at $30^{\circ} \mathrm{C}$ (Choe and Heu 1975). Brown and milled rice $\mathrm{ADV}$ s were graded from 1 to 7 , according to the standards set by the International Rice Research Institute (Table 1).

Analysis of QTLs associated with alkali digestion value

The genetic map consisting of 222 SSR markers was constructed by MAPMAKER/EXP Version 3.0 using the SNDH population (Lincoln et al. 1992). The completed genetic map used by the Plant Molecular Breeding Laboratory at the School of Applied Biosciences in Kyungpook National University. WINDOWS QTL Cartographer version 2.5 (WinQTLcart 2.5) was employed to analyze QTLs associated with the ADVs of brown and milled rice. The map was constructed with the Kosambi function, and the QTLs were detected by composite interval mapping (CIM) with a LOD (log of odds ratio) threshold of 3.0 (Zeng 1994).The standard QTL nomenclature was applied (McCouch et al. 1997).

\section{DNA extraction and PCR analysis for MAS}

To determine if the markers found from the QTL analysis are related to ADV, DNA extraction and PCR were performed using a NucleoSpin ${ }^{\circledR}$ Plant II Kit(MACHEREY-NAGEL, Cat. 740 770.250). In total, 13 varieties of the japonica type and seven varieties of the indica type were used for DNA extraction. To prepare the grains for extraction, $100 \mathrm{mg}$ brown rice was frozen with liquid nitrogen and then ground with mortar and pestle. The ground rice was placed into a $1.5 \mathrm{ml}$ tube, and $400 \mu \mathrm{L}$ P1 solution with $10 \mu \mathrm{L}$ RNase A were added. The tube was then placed in a $65^{\circ} \mathrm{C}$ water bath for 10 minutes. Subsequently, the rice solution was transferred into a NucleoSpin ${ }^{\circledR}$ Filter with collection tube and centrifuged at $13,000 \mathrm{rpm}$ for 2 minutes. Upon the end of the centrifuge cycle, $450 \mu \mathrm{L}$ PC solution was added into the collection tube and the contents were then transferred into a NucleoSpin ${ }^{\circledR}$ Plant II Column with a new collection tube. This was centrifuged at 13,000 rpm for 1 minute. The filtrate (in the spin column) was then removed and $400 \mu \mathrm{L}$ PW1 solution was added for washing. The sample solution was again centrifuged, at 13,000 rpm for 1 minute. $700 \mu \mathrm{L}$ PW2 solution was added to 
the filtrate, which was centrifuged at 13,000 rpm for 1 minute. To remove the washing solution from the filtrate and dry the silica membrane, $200 \mu \mathrm{L}$ PW2 solution was added and the solution centrifuged at 13,000 rpm for 2 minutes. The column containing the final filtrate was placed into a $1.5 \mathrm{ml}$ tube and $50 \mu \mathrm{L}$ PE solution was added. The sample solution was incubated at $65^{\circ} \mathrm{C}$ for 5 minutes and then centrifuged twice, for 1 minute at 13,000 rpm. Lastly, the DNA in the column was eluted by nuclease-free water.

PCR was conducted on the extracted DNA, with markers from the analysis of ADV-related QTLs. The $24 \mu \mathrm{L}$ PCR mixture consisted of $2 \mu \mathrm{L} 10-20 \mathrm{ng} / \mu \mathrm{L}$ template DNA, $1 \mu \mathrm{L}$ 5-10 pM of each primer, 0.1 $\mu \mathrm{L}$ Taq polymerase (Inclone Biotech Co., IN5001), $0.375 \mu \mathrm{L}$ dNTPs mixture, $2.4 \mu \mathrm{L}$ 10× Ex buffer, and $17.125 \mu \mathrm{L}$ nuclease-free water (QIAGEN, Cat. No.129114). PCR was conducted using a GeneAmp PCR System 2700 (Applied Biosystems, USA). The thermos cycling conditions were as follows: initial denaturation for 5 minutes at $96^{\circ} \mathrm{C}$, followed by 34 cycles of 30 seconds at $96^{\circ} \mathrm{C}, 30$ seconds at $55^{\circ} \mathrm{C}, 1$ minute at $72^{\circ} \mathrm{C}$, and a final extension for 8 minutes at $72^{\circ} \mathrm{C}$. The PCR products were stored at $4^{\circ} \mathrm{C}$ until needed. Amplicons were examined using gel electrophoresis (QIAGEN, QIAxcel) to determine suitability for subsequent genotyping.

\section{Results}

ADV evaluation was conducted in the both the crossed SNDH population and the parental cultivars, 'Cheongcheong' and 'Nagdong' (Table 2). DH brown rice ADVs were 1.87 \pm 1.01 and 2.58 \pm 1.23 of ADV in 2013 and 2014, respectively, while DH milled rice ADVs were $4.82 \pm 1.53$ and $4.93 \pm 0.92$ in 2013 and 2014, respectively. The broad sense heritability of ADVs score brown rice showed $70 \%$ and $83 \%$ in 2013 and 2014, respectively. Whereas, the broad sense heritability of milled rice showed 79\% and 85\% in 2013 and 2014. In the parental cultivars, 'Cheongcheong' (indica) and 'Nagdong' (japonica) brown rice ADVs were 1.96 and 1.68 in 2013, and 2.95 and 2.75 in 2014, respectively. In contrast, 'Cheongcheong' and 'Nagdong' milled rice ADVs were higher, at 5.61 and 4.10 in 2013, and 4.81 and 4.88 in 2014, respectively (Fig. 2).

In 2013, only five ADV-associated QTLs in milled rice were detected, on chromosomes 1, 3, 5 and 8. In 2014, four ADV-associated QTLs in milled rice were detected, on chromosome 5, 6 and only one ADV-associated QTL was found in brown rice, on chromosome 8 (Fig. 3). The milled rice and brown rice QTLs were named $\mathrm{qADM}$ and $\mathrm{qADB}$, respectively.

According to the 2013 results of the QTL analysis, QTLs qADM1-1 and qADM1-2 on chromosome 1 had LOD of 3.46 and 4.18, respectively. These two QTLs explained 0.31 and

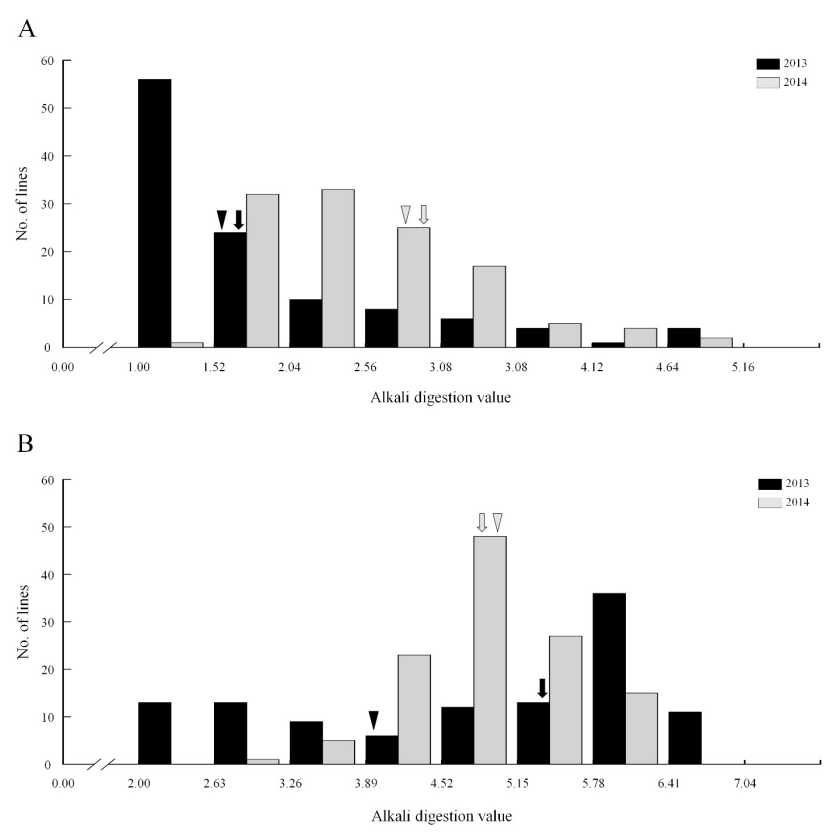

Fig. 2 Frequency distribution of the alkali digestion valuesin 2013 and 2014. The distribution of brown rice (A) and milled rice (B) are investigated for two years. The black bars and indicators represent 2013data while the white bars and indicators represent 2014 data. The arrows and triangles indicate Cheongcheong and Nagdong, respectively

Table 2 Alkali digestion values of $120 \mathrm{CNDH}$ (Cheongcheong/Nagdong doubled haploid) population and the parent cultivars

\begin{tabular}{ccccccccc}
\hline Variety & Year & Brown rice $^{\mathrm{a}}$ & Variance & $\mathrm{H}^{\mathrm{b}}(\%)$ & Milled rice $^{\mathrm{a}}$ & Variance $^{\mathrm{H}^{\mathrm{b}}(\%)}$ \\
\hline \multirow{2}{*}{ Cheongcheong } & 2013 & $1.96 \pm 0.76$ & 0.66 & - & $5.61 \pm 1.30$ & 0.25 & - \\
& 2014 & $2.95 \pm 0.14$ & 0.46 & - & $4.81 \pm 0.64$ & 0.04 & - \\
\multirow{2}{*}{ Nagdong } & 2013 & $1.68 \pm 0.81$ & 0.39 & - & $4.10 \pm 1.31$ & 2.41 & - \\
& 2014 & $2.75 \pm 0.15$ & 0.59 & - & $4.88 \pm 0.58$ & 0.16 & - \\
CNDH & 2013 & $1.87 \pm 1.01$ & 1.00 & 65 & $4.82 \pm 1.53$ & 0.51 & 86 \\
& 2014 & $2.58 \pm 1.23$ & 2.42 & 74 & $4.93 \pm 0.92$ & 0.40 & 83 \\
\hline
\end{tabular}

${ }^{\mathrm{a}}$ Mean $\pm \mathrm{SD}$, ${ }^{\mathrm{b}}$ Broad sense heritability 

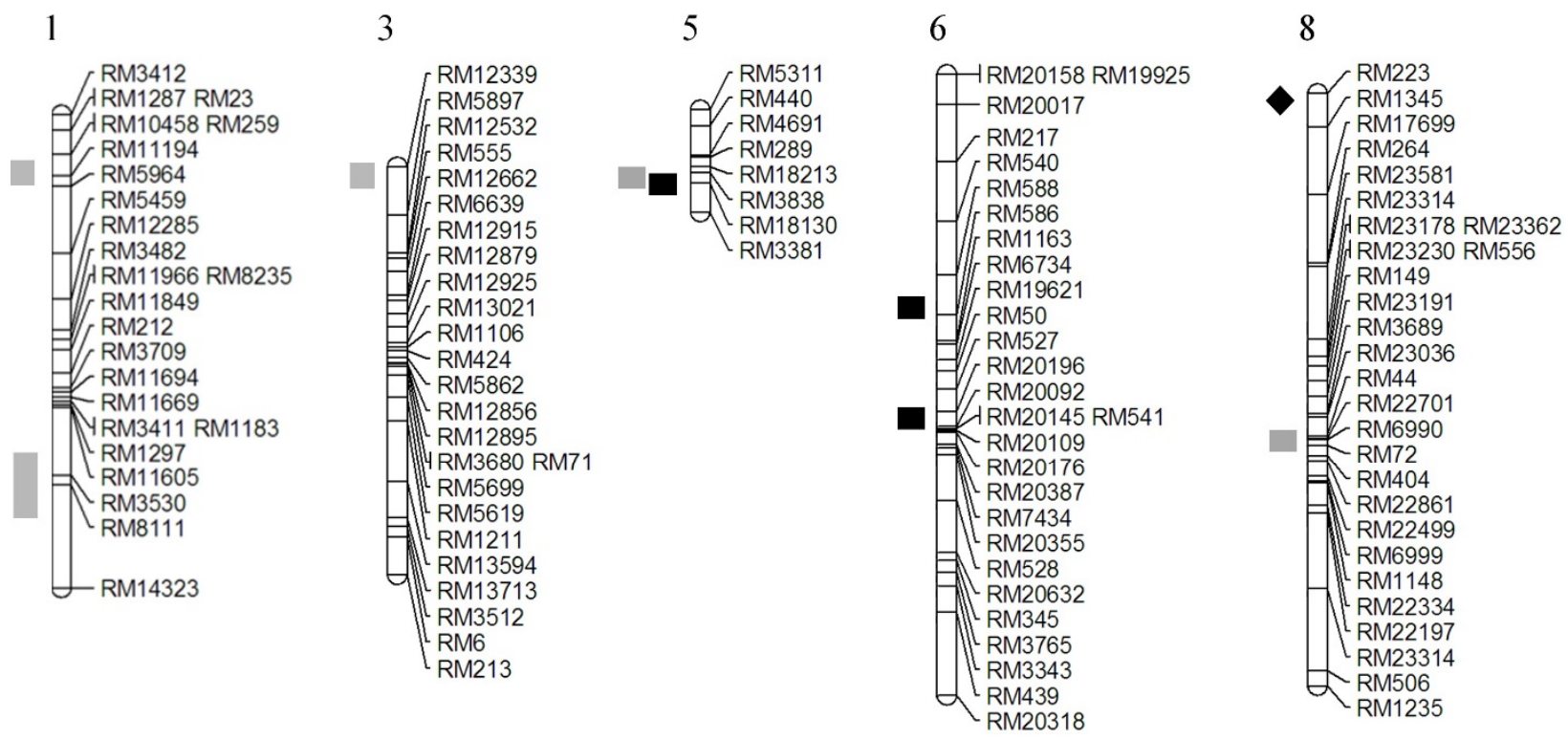

2013 Alkali digestion value of milled rice

2014 Alkali digestion value of milled rice

2014 Alkali digestion value of brown rice

Fig. 3 Genetic locations ofQTLs related to the alkali digestion of brown and milled rice. The gray quadrangles are QTLs for milled rice in 2013, while the back quadrangle and diamond are QTLs for milled rice and brown rice, respectively, in 2014

Table 3 QTL analysis for the alkali digestion value of brown and milled rice in DH population for two years

\begin{tabular}{|c|c|c|c|c|c|c|c|}
\hline Year & Chromosome & QTL & Region $^{a}$ & $\mathrm{LOD}^{\mathrm{b}}$ & Variation & Additive effect & Allele \\
\hline \multirow{5}{*}{2013} & 1 & qADM1-1 & RM10458-RM5964 & 3.46 & 0.31 & 0.86 & Cheongcheong \\
\hline & 1 & qADM1-2 & RM3530-RM8111 & 4.18 & 0.27 & -0.77 & Nagdong \\
\hline & 3 & qADM3 & RM14330- ${ }^{\mathrm{c}}$ & 3.69 & 0.27 & 0.67 & Cheongcheong \\
\hline & 5 & qADM5 & RM3838-18130 & 3.71 & 0.24 & 0.49 & Cheongcheong \\
\hline & 8 & qADM8 & RM44 & 3.15 & 0.34 & 0.44 & Cheongcheong \\
\hline \multirow{4}{*}{2014} & 5 & qADM5 & RM18130- & 3.75 & 0.29 & 0.22 & Cheongcheong \\
\hline & 6 & qADM6-1 & RM586- & 3.43 & 0.30 & 0.30 & Cheongcheong \\
\hline & 6 & qADM6-2 & RM20196-20092 & 3.25 & 0.36 & -0.23 & Nagdong \\
\hline & 8 & qADB8 & RM223- & 3.75 & 0.27 & 0.28 & Cheongcheong \\
\hline
\end{tabular}

${ }^{\mathrm{a}}$ The region of the QTLsinterval between markers, ${ }^{\mathrm{b}}$ Logarithm of the odds, ${ }^{\mathrm{c}}$ Only one marker detected.

0.27 of the phenotypic variation with additive effects of 0.86 and -0.77 , respectively. In other words, the qADM1-1 allele was from the 'Cheongcheong', while the qADM1-2 allele was from the 'Nagdong'. The QTL qADM3 was detected on chromosome 3 with an LOD of 3.69 and an additive effect of 0.67. This QTL explained 0.27 of the phenotypic variation and the qADM 3 allele was from the 'Cheongcheong'. The QTL qADM5 was detected on chromosome 5 with an LOD of 3.71 and an additive effect of 0.49 . This QTL explained 0.24 of the phenotypic variation and the qADM5 allele was from the 'Cheongcheong'. The QTL qADM8 was detected on chromosome 8 with an LOD of 3.15 and an additive effect of
0.44. This QTL explained 0.34 of the phenotypic variation and the qADM8 allele was from the 'Cheongcheong', too.

In the 2014 results, this QTL qADM5 was found on chromosome 5, with an LOD of 3.75 and an additive effect of 0.22 . The QTL explained 0.29 of the phenotypic variation and the qADM5 allele was from the 'Cheongcheong'. The QTL qADM6-1 and qADM6-2 on chromosome 6 had LOD of 3.43 and 3.75. Both an additive effect and the phenotypic variation explained $0.29,0.22,0.30$ and 0.30 , respectively. However, the qADM6-1 allele was from the 'Cheongcheong', the qADM6-2 allele was from the 'Nagdong'. Finally, the QTL qADB8 was detected on chromosome 8 with an LOD of 3.75 , 


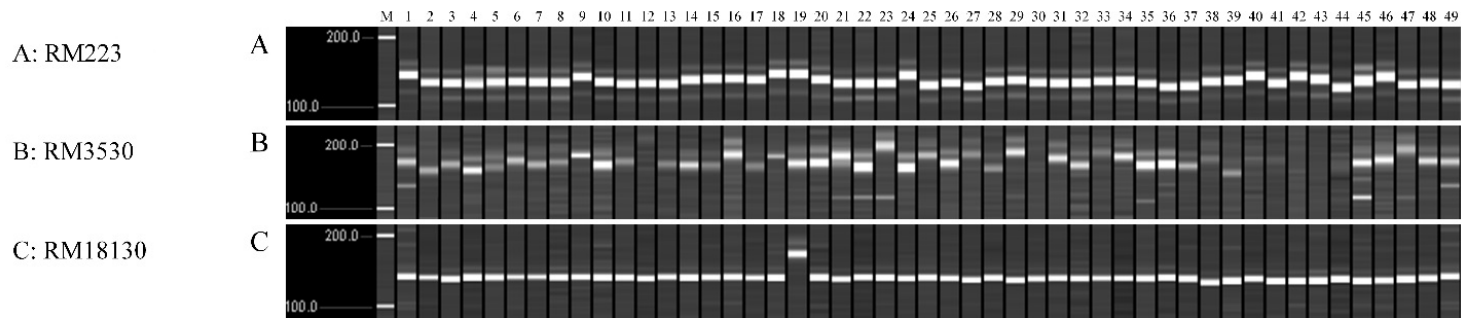

Fig. 4 The PCR results of 49cultivars using three markers detected from the analysis of QTL related to alkali digestion value. A: RM223, B: RM3530, C: RM18130, 1: Cheongcheong, 2: Nagdong, 3: Jogwang, 4: Dongjin 1, 5: Dunnae, 6: Keumo 3, 7: Goun, 8: Geuroo, 9: Haepyeong, 10: Hanareum, 11: Hopum, 12: Hwayoung, 13:Jeoktomi, 14: Jinbu, 15: Jinmi, 16: Joan, 17: Joun, 18: Junam, 19: Namcheon, 20: Namil, 21:Nampyeong, 22: Obong, 23: Odae, 24: Samdeog, 25: Samgang, 26: Sangju, 27: Seolgang, 28: Wangchal, 29: Yangjo, 30: U-2, 31: Akenohgshi, 32: Fukunohana, 33: Hokuriku 130, 34: Ishikari, 35: Kitaake, 36: Koganenilcari, 37: Milk Princess, 38: Milky queen, 39: Princess sari, 40: Silewah, 41: Cakmak, 42: Demir, 43: Ece, 44: Efe, 45: Gonen, 46: Karadeniz, 47: Kizilmak, 48: Ilmi, 49: Baekjinju.

an additive effect of 0.28 and the phenotypic variation of 0.27 , respectively. And the qADB8 allele was from the 'Cheongcheong', too (Table 3).

The polymorphisms found 49 varieties of japonica and indica types, including the 'Cheongcheong' and 'Nagdong' parent cultivars, were analyzed using three markers from the detected QTLs (Fig. 4). Nine cultivars (9, 18, 19, 24, 40, 42, $43,45,46)$ shown such as indica type. The results show that genetic polymorphisms in these varieties can be detected using the markers. Therefore, we considered the two markers, RM223, to be suitable for marker-assisted selection. But RM3530 and RM18130 were not distinguish in electrophoresis and more need study for marker in this study.

\section{Discussion}

A previous study examining heritability values were high in ADV treatment for eating quality (Kim et al. 1988). QTLs related to rice $\mathrm{ADVs}$ found that the phenotypic variation explained by the QTL on chromosome 8 was $48 \%$ (Lee et al. 2000). Thus, the QTLs associated with CNDH brown and milled rice found in this study are minor, because the total phenotypic variation explained by all QTLs in this study is close to the phenotypic variation explained by two QTLs found on chromosome 3 and 11 by Lee et al. (2000), which is only half of the QTLs they detected. The fact that so many major and minor QTLs are present is unsurprising, as previous studies have demonstrated that $\mathrm{ADV}$ is clearly a very polygenic trait. For example, Heu and Park (1979) reported that the major genes affecting ADV variation is influenced by several modifying genes, and differing expression of governing genes also affect their influence on ADV. Furthermore, a lower $\mathrm{ADV}$ was found to be the dominant trait.

Our results, showing ADV-related QTLs on chromosomes
1, 3, 5, 6, and 8, corroborate previous research. For instance, Shin et al. (1995) found five such QTLs on chromosome 1, 5 , 7, 8, and 12. Moreover, QTLs associated with two similar traits, the alkali spreading value and the alkali-spreading score (related to gelatinization temperature), were mapped to chromosomes 2, 3, and 6 (Yan et al. 2001) and chromosome 6 (Sabouri et al. 2012, Aluko et al. 2004), respectively. In a large-scale study comparing grain quality characters that spanned multiple rice strains, the amylose content of indica rice was positively correlated to alkali spreading value and negatively correlated to gel consistency (Wang et al. 2005). However, no significant correlations were found between amylose content, alkali spreading value, and gel consistency in japonica rice (Wang et al. 2005). Additionally, a gene influencing the alkali-spreading score (alk) was found to be linked to the Wx locus (McKenzie and Rutger 1983; Sano 1984). Rice grain gelatinization temperature is one of the most important determinants of cooking quality. Generally, this trait is tested indirectly using the alkali spreading value test (Little et al. 1958), which is related to the alkali digestion value used in this study (Table 1). The 'Cheongcheong' and 'Nagdong' ADVs exhibited only small differences, but greater differences were observed between brown rice and milled rice. This is because the $\mathrm{KOH}$ solution can more easily reach amylose in milled rice compared to brown rice, which is shielded by a bran layer. Thermal properties closely is linked to alk identified as a major gene in a research and the QTLs on chromosome 1 and 7 showed different effects to the alk gene. In this study, we detected three ADV-related QTLs across two years. As stated earlier, our results supported findings from prior studies: two QTLs (qADM) on chromosome 1 of milled rice had been detected previously (Bao et al. 2004), and three QTLs on chromosome 5, 6 and 8 had been reported by Kang et al. (1996). We point out, however, that although the QTLs reported here are similar to those reported in previous research 
in terms of chromosomal position, the precise gene locations remain unknown. Thus, future research should focus on the fine mapping of QTLs detected with a single marker.

Differing out comes across studies may be due to variation in study materials and study environment (Xiao et al. 1996). We analyzed polymorphisms in 49 rice varieties, including indica- and japonica-type cultivars, based on three markers detected from the QTL analysis. We consider this marker useful for marker-assisted selection in breeding programs to improve the eating quality of cooked rice.

\section{Acknowledgments}

This work was supported by a grant from the Next-Generation BioGreen21 Program(No. PJ011257012016), Rural Development Administration, Republic of Korea.

\section{References}

Aluko G, Martinez C, Tohme J, Castano C, Bergman C, Oard JH (2004) QTL mapping of grain quality traits from the interspecific cross Oryza sativa $\times$ O. glaberrima. Theor Appl Genet 109:630-639

Bao J, Sun M, Zhu L, Corke H (2004) Analysis of quantitative trait loci for some starch properties of rice (Oryza sativa L.), thermal properties, gel texture and swelling volume. J Cereal Sci 39:379-385

Choe ZR, Heu MH(1975) Optimum conductions for alkali digestivity test in rice. Korean J Crop Sci 19:7-13

Heu MH, Park SZ (1979) Genetic behavior of alkali digestibility in rice endosperm. Alkali digestibility of hybrid seed and $\mathrm{F}_{2}$ grains in the crosses between low and high ADV parents. Korean J Breed 11:196-200

Julian'o BO (1985) Criteria and tests for rice grain qualities. In rice, Chemistry and Technology. AACC, USA, 443-524

Juliano BO (2003) Rice chemistry and quality. Philippine Rice 7. Research Institute. 215-220

Juliano BO (1985) Rice, Chemistry and Technology 2nded. AACC

Kang HJ, Cho YG, Lee YT, Kim YD, Eun MY, Shim JU (1998) QTL mapping of genes related with grain chemical properties based on molecular map of rice. Korean J Crop Sci 43:199-204

Kim HT, Park GH, Kim DU (1988) Genetic Studies on Grain Shape and Quality in Rice - II. Genetic Analysis of Chalkiness and Alkali Digestibility Value. Agric. Res.Bull.Kyungpook Natl Univ 6:31-36
Kwak TS (2009) Variation of amylose, Protein, alkali digestion value, and moisture contents in rice varietal groups classified by the days from seedling to heading. Korean J Intl Agri 21:28-32

Kwak TS, Yeo JH, Eun MY, Cha YS (2004) QTL for quality properties in the Milyang23 x Gyhobyeo recombinant inbred lines by different locations. Korean J Crop Sci 449:539-545

Lee JH, Cho YS, Jung KH, Yang SJ, Hwang HG, Kim NS, Choi HC (2000) Analysis of quantitative trait Loci (QTL) related to rice alkali digestion value and amylose content. Korean J Genetics 22:71-79

Lincoln S, Daley M, Lander E (1992) Constructing Genetic Maps with APMAKER/EXP 3.0. Whitehead InstituteTechnical Report, 3rd Edition

Little RR, Hilder GB, Dawson EH, Elsie H (1958) Differential effect of dilute alkali on 25 varieties of milled milled rice. Cereal Chemistry 35:111-126

McCouch SR, Cho YG, Yano M, Paul E, Blinstrub M, Morishima H, Kinoshita T (1997) Report on QTLnomenclature. Rice Genet Newsl 14:11-131

Mckenzie KS, Rutger JN (1983) Genetic analysis of amylose content, alkali spreading score, and grain dimensions in rice. Crop Sci 23:306-313

He P, Li SG, Qian Q, Ma QY, Li JZ, Wang WM, Chen Y, Zhu LH (1999) Genetic analysis of rice grain quality. Theor Appl Genet 92:502-508

Hu P, Zhao H, Duan Z, Linlin Z, Wu D (2004) Starch digestibility and the estimated glycemic score of different types of rice differing in amylose contents. J Cereal Sci 40:231-237

Sano Y (1984) Differential regulation of waxy gene expression in rice endosperm. Theor Appl Genet 68:467-473

Sabouri A, Rabiei B, Toorchi M, Aharizad S, Moumeni A (2012) Mapping quantitative trait loci (QTL) associated with cooking quality in rice (Oryza sativa L.). AJCS6, 808-814.

Shi CH, Zhu J, Zang RC, Chen GL (1997) Genetic and heterosis analysis for cooking quality traits of indica rice in different environments. Theor. Appl. Genet. 95:294-300

Wang D, Zhang X, Zhu Z, Chen N, Min J, Yao Q, Yan J, Liao X (2005) Correlation analysis of rice grain quality characteristics. Zuowuxuebao 31:1086-1091

Xiao J, Li J, Yuan L, Iwata N (1996) Identification of QTLs affecting traits of agronmic in a recombinant inbred population derived from a subspecific rice cross. Theor. Appl. Genet. 92:260-244

Yan CJ, Xu CW, Yi CD, Liang GH, Zhu LH, Gu MH (2001) Genetic analysis of gelatinization temperature in rice via microsatellite (SSR) markers.Acta genet.Sinica 28:1006-1011

Zeng ZB (1994) Precision mapping of quantitative trait loci. Genetics 136:1457-68 